\title{
Using UEM to Probe Buried Structures in Real Space: An Analog to Phonon Spectroscopy
}

\author{
Elisah VandenBussche and David Flannigan
}

University of Minnesota, Minneapolis, Minnesota, United States

The process by which photoexcited semiconductors return to the ground state consists of a series of strongly-correlated, many-body interactions which overlap in space and time. Such behaviors have both fundamental and practical implications, which include insights into the quantum nature of matter and control of device and materials behaviors in electronic and optoelectronic applications. Accordingly, extensive work has been done to illuminate the sequence of hot-carrier excitation, carrier-carrier scattering, carrier-lattice scattering and thermalization, recombination, and lattice heat diffusion [13]. Linked to carrier-driven dynamics is the excitation of coherent acoustic phonons (CAPs). Because the behavior of these collective lattice oscillations depends on the structural and electronic properties of the material through which they propagate, CAPs can be used as an intrinsic, multi-faceted characterization tool. Indeed, CAP spectroscopy, in which transient reflectivity measurements are used to track CAPs as they travel into a material, has been used to access depth-dependent structural properties of materials and buried interfaces [4]. In addition to all-optical methods, ultrafast scattering techniques are well-suited for the study of CAP behaviors owing to the associated lattice distortions. For example, CAPs have been directly imaged with ultrafast electron microscopy (UEM), and the ability to spatiotemporally resolve discrete phonon behaviors, such as phase velocity and time-varying dispersion, has been demonstrated [5]. While CAP spectroscopy benefits from 10-nm spatial resolution in the $z-$ direction, there are many systems where the structural or optoelectronic properties under investigation vary in plane, warranting additional study with high spatial resolution in the $x y$ plane. It is possible, then, to imagine the utility of probing in-plane CAP behaviors with the high spatiotemporal resolutions possible with UEM, thus enabling real-space inference of spatially heterogeneous structural and optoelectronic properties via correlation with time-domain phonon behaviors.

Here, we demonstrate the sensitivity of CAP phase velocity to changes in atomic structure, as measured with UEM imaging. The model system used was a GaAs specimen [Figure 1(a)] with a sandwich-structure morphology, where the largest exposed faces showed significant amorphization due to ion-beam damage incurred during specimen preparation. After UEM measurement of photoexcited CAPs in the amorphized specimen, the sandwich structure was photothermally annealed in situ, which increased the degree of crystallinity and produced an associated hardening of the specimen. Following this, the photoexcited CAP behaviors were again imaged and quantified. Space-time contour-plot (STCP) analyses were completed for both experiments, where 3D data (two spatial dimensions plus time) is collapsed into a single 2D frame in order to capture CAP behavior through time, as shown in Figure 1(b). This enables tracking of individual phonon movement with few-pixel (approximately 10-nm) precision and measurement of phase velocities with average uncertainties of $0.1 \mathrm{~nm} / \mathrm{ps}$. The result of the STCP analysis is the time-resolved measurement of hypersonic CAPs which decay to the range of longitudinal speeds of sound in both the pre-annealed and the post-annealed material. Owing to variations in elastic constants, sound velocities in the amorphized and relatively crystalline specimens will vary, thus producing a difference in asymptotic values of the phase velocities. Indeed, the results summarized in Figure 1(c) demonstrate the sensitivity of UEM imaging to such a behavior. As can be seen, the post-annealed specimen (with higher degree of crystallinity) displays a higher phase-velocity asymptotic value, which is consistent with the expected hardening of the specimen following annealing. Additionally, both asymptotic velocities are closer to the expected speed of sound for a crystalline specimen than for an amorphous specimen, as is expected for 
amorphized regions which retain some crystalline order rather than becoming fully disordered from ionbeam damage. These observations based on quantitative measurement of CAPs with UEM demonstrate the sensitivity of this technique to atomic structural changes in semiconducting materials, facilitating future work in using UEM to access spatially-resolved information about buried structures and optoelectronic properties via high-resolution, real-space measurements [6].
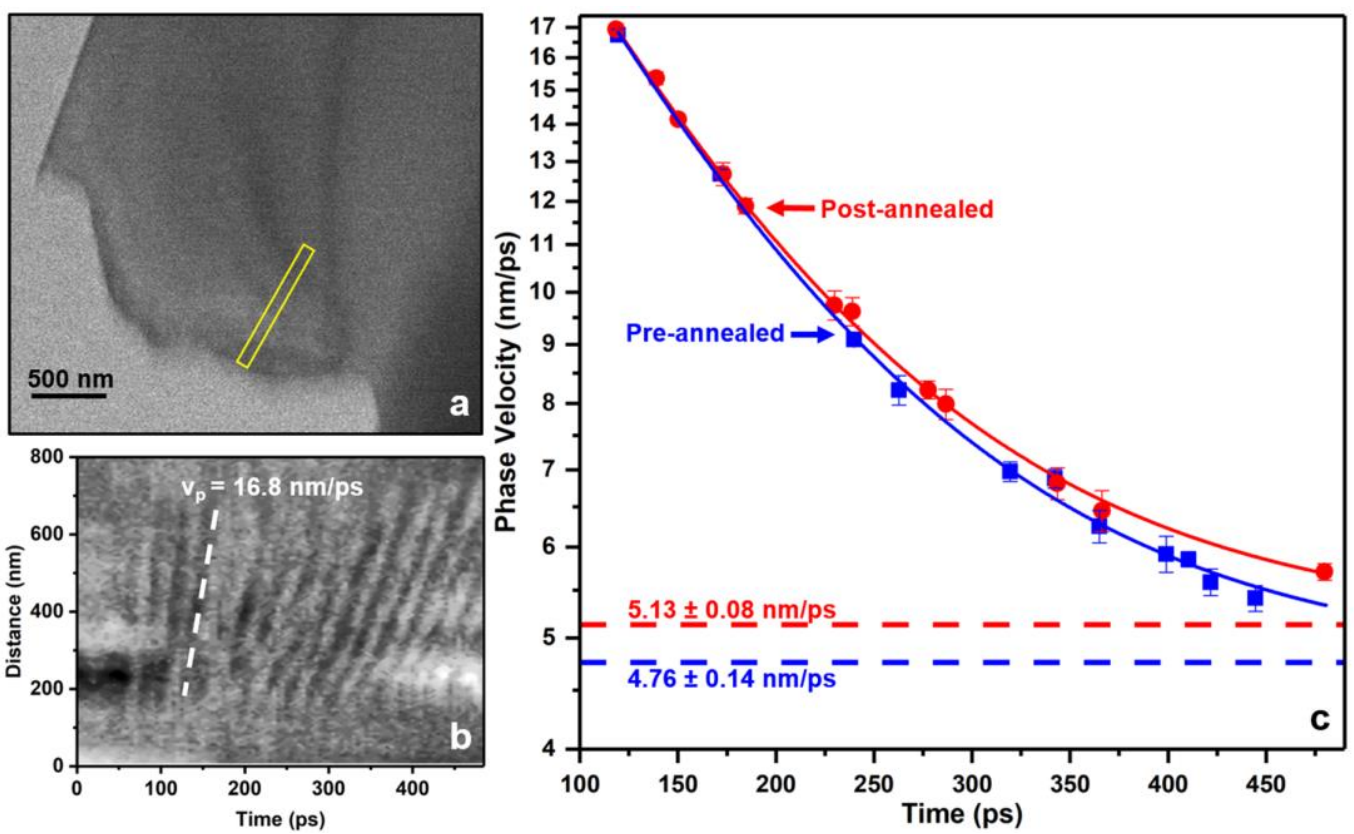

Figure 1. Analog to CAP spectroscopy with UEM. (a) Select UEM image with ROI indicated (yellow rectangle), from which the STCP shown in (b) was generated. (b) STCP spanning from $\mathrm{t}=-5 \mathrm{ps}$ to $\mathrm{t}=$ 485 ps. Each dark band is a phonon wavefront (the position of a select wavefront is indicated with the white dashed line). The phase velocity of the marked wavefront is $16.8 \mathrm{~nm} / \mathrm{ps}$. (c) Time-dependent phase velocity for pre- and post-annealed GaAs (blue squares and red dots, respectively). The data are fit (solid curves) with a single exponential decay function, and the asymptotes for each curve are indicated with horizontal, colored dashed lines. The error bars on the data points are one standard deviation of the fits to the associated space-time contour plots, and errors on the asymptotic values are one standard error of fits to the phase velocity data.

\section{References}

[1] A. Othonos, J. Appl. Phys. 83, (1998), 1789.

[2] D. Von Der Linde, et al., Phys. Rev. Lett. 44, (1980), 1505.

[3] J. A. Kash, et al., Phys. Rev. Lett. 54, (1985), 2151.

[4] V. E. Gusev and P. Ruello, Appl. Phys. Rev. 5, (2018), 031101.

[5] D. R. Cremons, et al., Phys. Rev. Mater. 1, (2017), 073801.

[6] This material is based on work supported by the U.S. Department of Energy, Office of Science, Office of Basic Energy Sciences under Award No. DE-SC0018204. This material is based upon work supported by the National Science Foundation Graduate Research Fellowship Program under Grant No. DGE1348264. Part of this work was carried out in the College of Science and Engineering Characterization Facility, University of Minnesota, which has received capital equipment funding from the NSF through the UMN MRSEC program under Award Numbers DMR-0819885 and DMR-1420013. 